\title{
Nanoscale
}

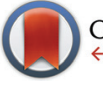

CrossMark

\& click for updates

Cite this: Nanoscale, 2015, 7, 4361

Received 17th December 2014,

Accepted 28th January 2015

DOI: $10.1039 / c 4 n r 07458 \mathrm{k}$

www.rsc.org/nanoscale

\section{Reduced ceria nanofilms from structure prediction $\uparrow$}

\author{
Sergey M. Kozlov, ${ }^{a}$ Ilker Demiroglu, $\stackrel{t}{a}^{a}$ Konstantin M. Neyman ${ }^{\star a, b}$ and \\ Stefan T. Bromley*a,b
}

\begin{abstract}
Experimentally, $\mathrm{Ce}_{2} \mathrm{O}_{3}$ films are used to study cerium oxide in its fully or partially reduced state, as present in many applications. We have explored the space of low energy $\mathrm{Ce}_{2} \mathrm{O}_{3}$ nanofilms using structure prediction and density functional calculations, yielding more than $\mathbf{3 0}$ distinct nanofilm structures. First, our results help to rationalize the roles of thermodynamics and kinetics in the preparation of reduced ceria nanofilms with different bulk crystalline structures (e.g. A-type or bixbyite) depending on the support used. Second, we predict a novel, as yet experimentally unresolved, nanofilm which has a structure that does not correspond to any previously reported bulk $A_{2} B_{3}$ phase and which has an energetic stability between that of A-type and bixbyite. To assist identification and fabrication of this new $\mathrm{Ce}_{2} \mathrm{O}_{3}$ nanofilm we calculate some observable properties and propose supports for its epitaxial growth.
\end{abstract}

Cerium oxide (ceria) may gradually change its stoichiometry between $\mathrm{CeO}_{2}$ and $\mathrm{Ce}_{2} \mathrm{O}_{3}$ depending on the environment. This makes it a key reducible oxide in numerous technological applications (e.g. gas sensors, fuel cells, catalysis). ${ }^{1-3}$ Nanostructuring dramatically affects the reducibility of $\mathrm{CeO}_{2}$, facilitating the formation of $\mathrm{O}$ vacancies. ${ }^{4-6}$ The latter results in the increased catalytic activity of noble metals supported on $\mathrm{CeO}_{2}$ and, in particular, on nanostructured $\mathrm{CeO}_{2} \cdot{ }^{1,7}$ Since stoichiometric cerium dioxide is present only under oxidizing conditions and/or at moderate temperatures, there is growing interest in (partially) reduced forms of ceria. Recently, ultrathin supported nanoscale films of crystalline stoichiometric cerium sesquioxide $\mathrm{Ce}_{2} \mathrm{O}_{3}$ have been prepared on various substrates. ${ }^{8,9}$ Such nanofilms provide well-defined model systems for studying the reactivity of ceria under extremely reducing

\footnotetext{
${ }^{a}$ Departament de Quimica Física and Institut de Química Teòrica i Computacional (IQTCUB), Universitat de Barcelona, 08028 Barcelona, Spain

${ }^{b}$ Institució Catalana de Recerca i Estudis Avançats (ICREA), 08010 Barcelona, Spain. E-mail:konstantin.neyman@icrea.cat, s.bromley@ub.edu

$\dagger$ Electronic supplementary information (ESI) available: Graph of IP versus DFT relative energies for nanofilms, GGA $+U$ calculated lattice parameters and atomic coordinates of NF1-4 nanofilms. See DOI: 10.1039/c4nr07458k

$\$$ Current address: School of Chemistry, University of Birmingham, Edgbaston, Birmingham, B15 2TT, UK
}

conditions $^{8}$ and have potential applications as high- $k$ transistor gate dielectrics. ${ }^{9}$ Curiously, many of these nanofilms do not possess the hexagonal A-type structure, which is generally thought to be the thermodynamically stable bulk $\mathrm{Ce}_{2} \mathrm{O}_{3}$ polymorph.

Generally, for many oxide materials reduction of their thickness to only a few monolayers has opened up a wealth of new technological opportunities in diverse application areas. ${ }^{10}$ In only a few cases, however, are supported oxide nanofilms found to possess well-ordered atomic structures unlike that of the corresponding most stable bulk crystalline phase (e.g. $\left.\mathrm{Al}_{2} \mathrm{O}_{3},{ }^{11} \mathrm{SiO}_{2},{ }^{12,13} \mathrm{MgO},{ }^{14} \mathrm{ZnO}^{15}\right)$. These nanofilms can be divided into two types: (i) non-stoichiometric films (e.g. oxides of $\mathrm{Al}^{11} \mathrm{Si}^{12}$ ), where chemical bonds of a noticeable strength form with a strongly interacting support, or (ii) stoichiometric films, essentially without chemical bonds with the support (e.g. $\mathrm{MgO},{ }^{14} \mathrm{ZnO},{ }^{15} \mathrm{SiO}_{2}{ }^{13}$ ). In all these cases ab initio calculations have been indispensable in confirming, ${ }^{11,12,16}$ and even predicting ${ }^{17}$ the atomic structure of the nanofilms. Although, in a real experimental set-up, oxide nanofilms are almost always grown on a support, computationally, via modelling free-standing sheets, one can enquire into the inherent stability of different nanofilm structures independently of a specific support. For known stoichiometric oxide nanofilms, the interactions with the support tend to be weak and the nanofilm structures can be well-described by free-standing sheet models. ${ }^{16-18}$ Note that even for nanofilms that weakly interact with the support epitaxial matching between the nanofilm and the support is generally observed. Comparison of free-standing models with experimental data can help to determine to what extent the observed polymorph is the result of: (i) intrinsic nanoscale structural/energetic tendencies of the material or (ii) experimental conditions (e.g. epitaxy with a specific support, metastability of obtained structures, etc.).

Herein we use a powerful structure search method and accurate electronic structure calculations to systematically explore the stabilities and structures of a range of free-standing stoichiometric $\mathrm{Ce}_{2} \mathrm{O}_{3}$ nanofilms in order to understand the experimental observations. Specifically, we address the issue of thermodynamic versus kinetic stability in experimentally 
prepared $\mathrm{Ce}_{2} \mathrm{O}_{3}$ nanofilms. Moreover, we predict new low energy $\mathrm{Ce}_{2} \mathrm{O}_{3}$ nanofilms that may be prepared in the future.

Diminution of inorganic materials to the nanoscale often induces one or more alternative atomic orderings relative to the most stable bulk crystal. ${ }^{19}$ In order to test this possibility for reduced ceria we explored the space of stable $\mathrm{Ce}_{2} \mathrm{O}_{3}$ nanofilm structures with $\sim 1 \mathrm{~nm}$ thickness, i.e. containing four monolayers (MLs). Here, we define monolayers based on the number of cerium atoms, i.e. the $\mathrm{O}-\mathrm{Ce}-\mathrm{O}-\mathrm{Ce}-\mathrm{O}$ unit found in the vertical stacking of atomic layers in A-type $\mathrm{Ce}_{2} \mathrm{O}_{3}(001)$ is counted as $2 \mathrm{ML}$. We employed the simulated mechanical annealing (SMA) technique ${ }^{20-22}$ for searching the space of low energy film structures. Following the experimental observation of structural relaxation via application of mechanical stress (termed as mechanical annealing ${ }^{23}$ ) in submicrometre atomic systems, the SMA method consists of cyclically gradually compressing and stretching the simulated $\mathrm{Ce}_{2} \mathrm{O}_{3}$ nanofilms laterally (by up to $\pm 30 \%$ ) in a step-wise fashion. After each application of stress/strain to the nanofilm structure (achieved through systematically varying the cell parameters) all atomic positions are optimised. Upon these optimisations the atomic positions sometimes relaxed to give a new polymorph. We repeatedly applied the SMA stretching and compressing procedure to the $\mathrm{Ce}_{2} \mathrm{O}_{3}$ nanofilms for every new polymorphic structure found until no more new structures could be found. To reduce the bias on the choice of the initial nanofilm structure, we repeated the above process starting from three distinct archetypal $\mathrm{A}_{2} \mathrm{O}_{3}$ sesquioxide polymorphs: corundum, A-type, and bixbyite. Due to the high computational cost of following this protocol directly with $a b$ initio methods, we initially performed the SMA search with suitable classical interatomic potentials (IPs) ${ }^{24-26}$ using the GULP ${ }^{27}$ code. From this search, ten of the resulting lowest energy nanofilm structures were then optimised using density functional theory (DFT) calculations. As detailed below (see also ESI, Fig. S1 and S2 $\dagger$ ) both the lattice parameters and the relative energies calculated using IPs and DFT schemes correlate with one another very well. This excellent correspondence is in line with our previous experience in modelling stoichiometric ${ }^{4,28}$ and reduced ceria nanoparticles, ${ }^{26}$ giving us confidence in using the IPs for our SMA searches.

In all reported periodic DFT calculations for both nanofilm and bulk structures, the unit cell parameters and all atomic positions were locally optimized (forces $<0.2 \mathrm{eV} \mathrm{nm}^{-1}$ ) with the PW $91^{29}$ form of the generalized gradient approximation (GGA) functional using the VASP code. ${ }^{30}$ An onsite Coulombic correction $\left(U_{\text {eff }}=U-J\right)^{31,32}$ was applied to obtain a localized description of $\mathrm{Ce} 4 \mathrm{f}$-electrons, resulting in a GGA $+U$ corrected functional. Following previous studies, ${ }^{4,5}$ a $U_{\text {eff }}$ value of $4 \mathrm{eV}$ was used. The suggestion that a LDA $+U$ description of the relative stabilities of $\mathrm{Ce}_{2} \mathrm{O}_{3}$ polymorphs may provide a better match to experiment than a GGA $+U$ approach $^{33}$ is briefly discussed below. The projector augmented wave approach ${ }^{34,35}$ was used to describe the effect of core electrons on valence states, with the latter represented by a plane wave basis with a $600 \mathrm{eV}$ cut-off. Nanofilms were separated by over $1 \mathrm{~nm}$ in the $c$-stacking direction to avoid spurious periodic interactions. Reciprocal space $k$-point sampling was achieved through appropriate Monkhorst-Pack grids ${ }^{36}$ (see Table 1). Tests showed that all nanofilm energies were converged to $<0.5 \mathrm{~kJ}$ $\mathrm{mol}^{-1}$ per $\mathrm{Ce}_{2} \mathrm{O}_{3}$ with respect to $k$-point sets and completeness of the plane wave basis.

\section{Bulk calculations}

The hexagonal A-type phase is generally thought to be the most thermodynamically stable bulk phase of $\mathrm{Ce}_{2} \mathrm{O}_{3} \cdot{ }^{37}$ Our GGA $+U$ calculations, however, predict the A-type structure to be higher in energy than the cubic bixbyite structure $\left(E_{\mathrm{rel}}=\right.$ $19.9 \mathrm{~kJ} \mathrm{~mol}^{-1}$ per $\mathrm{Ce}_{2} \mathrm{O}_{3}$, see Table 1). Ref. 33 reported that the A-type polymorph becomes more energetically stable than the bixbyite structure when an LDA $+U$ scheme is used instead of a GGA $+U$ treatment. Our DFT calculations using the local density approximation with a Hubbard $U$ correction (LDA $+U$, with $U=6 \mathrm{eV}$ ) bring the energies of the two phases closer whereby the A-type phase becomes only $0.5 \mathrm{~kJ} \mathrm{~mol}^{-1}$ per $\mathrm{Ce}_{2} \mathrm{O}_{3}$ less stable than bixbyite. We note that the lower relative energetic stability of bixbyite in ref. 33 can be probably ascribed to the known problem of the presence of many meta-stable selfconsistent electronic solutions to Kohn-Sham equations for reduced cerium oxide. These solutions differ by the shape and symmetry of the occupied f-orbitals of $\mathrm{Ce}^{3+}$ cations, which may be sub-optimal in the electrostatic Madelung potential of the crystal. $^{38}$ We found that, for bixbyite especially, occupied forbitals would often converge to be $\mathrm{f}_{z^{3}}$-like, instead of the more stable $\mathrm{f}_{x y z}$-like ones, significantly affecting the calculated total energy of the system. ${ }^{39}$ In general, due to their more refined account of electron density variations, GGA functionals have proven to be superior to LDA functionals for calculating the relative stability of different oxide polymorphs when the coordination environment of the constituent atoms varies (e.g. $\left.\mathrm{SiO}_{2},{ }^{40} \mathrm{HfO}_{2}{ }^{41}\right)$. Specifically, GGA functionals help to correct the tendency of LDA functionals to overstabilise polymorphic

Table 1 In-plane film lattice parameter ( $a_{0}$ in pm), relative energies $\left(E_{\text {rel, }}\right.$ with respect to bixbyite, per $\mathrm{Ce}_{2} \mathrm{O}_{3}$ unit, in $\mathrm{kJ} \mathrm{mol}^{-1}$ ), MonkhorstPack k-point mesh, and thicknesses (in pm) of optimised $\mathrm{Ce}_{2} \mathrm{O}_{3}$ bulk polymorphs and nanofilms from GGA $+U(\mathrm{PW} 91+4 \mathrm{eV})$ calculations

\begin{tabular}{llrlll}
\hline System & $a_{0}$ & $a_{0}$ per unit & $E_{\text {rel }}$ & $k$-points & Thick \\
\hline Bulk & & & & & \\
A type & $391^{a}$ & 391 & 19.9 & $5 \times 5 \times 5$ & $\infty$ \\
Bixbyite & 1130 & 399 & 0 & $3 \times 3 \times 3$ & $\infty$ \\
& & & & & \\
Film & & & & & \\
A type & 384 & 384 & 11.1 & $3 \times 3 \times 1$ & 1081 \\
Bixbyite & 1587 & 397 & 0 & $1 \times 1 \times 1$ & 1186 \\
NF1 & $713 \times 707^{b}$ & $\sim 410$ & 5.5 & $5 \times 5 \times 1$ & 1129 \\
NF2 & 1364 & 394 & 20.8 & $3 \times 3 \times 1$ & 1147 \\
NF3 & $1383 \times 1379^{b}$ & $\sim 399$ & 26.2 & $3 \times 3 \times 1$ & 1150 \\
NF4 & 1407 & 406 & 22.2 & $3 \times 3 \times 1$ & 1118
\end{tabular}

${ }^{a}$ The experimental value is $389 \mathrm{pm} .{ }^{37}{ }^{b}$ Two lattice parameters are given for films with a distorted hexagonal structure. 
structures that have more bonds per atom. In the present study when going from bixbyite to A-type, the average bonding coordination environment of Ce increases from six to seven; this may rationalize the increased relative stabilization of Atype in $\mathrm{LDA}+U$ calculations with respect to GGA $+U$ treatments. The advantage of a GGA-based approach over LDA is expected to be more pronounced for energies of structures with less homogeneous electron densities, in situations where bonds are being stretched, or for non fully periodic structures.

Although in the case of the relative bulk energetics of bixbyite versus A-type polymorphs, GGA $+U$ appears to overcompensate the failings of LDA $+U$; in principle GGA $+U$ should provide an improved energetic description of $\mathrm{Ce}_{2} \mathrm{O}_{3}$ systems. One way to assess this assertion is to compare the GGA $+U$ results with those from computationally intensive calculations employing hybrid functionals, the current DFT benchmark standard for periodic systems like ceria. ${ }^{3}$ Using the hybrid HSE06 functional, ${ }^{42}$ we find bixbyite to be more stable than Atype by $25 \mathrm{~kJ} \mathrm{~mol}^{-1}$ per $\mathrm{Ce}_{2} \mathrm{O}_{3}$ unit, confirming the energetic ordering calculated using the GGA $+U$ approach. Considering the above mentioned arguments, in this work focused on the calculated properties of strained surface-terminated nanostructures, which possess novel polymorphic structures with variable bonding coordination, we preferred GGA $+U$ over LDA $+U$.

\section{Nanofilm calculations}

The strain versus total energy curves resulting from the SMA searches for low energy $\mathrm{Ce}_{2} \mathrm{O}_{3}$ four ML nanofilms using IPs are shown in Fig. 1a. These searches revealed more than 30 distinct nanofilm structures of which ten with the lowest energy were further optimized using DFT calculations. The results for six of them, A-type, bixbyite and nanofilms 1 to 4 (NF1-NF4), as well as for A-type and bixbyite bulks are presented in Table 1. Bixbyite, as a four ML nanofilm, is still predicted by our GGA + $U$ calculations to be more stable than the corresponding A-type nanofilm. However, its stability with respect to A-type decreases to $11.1 \mathrm{~kJ} \mathrm{~mol}^{-1}$ from $19.9 \mathrm{~kJ} \mathrm{~mol}^{-1}$ per $\mathrm{Ce}_{2} \mathrm{O}_{3}$ in the bulk. This reduction in polymorphic energy differences when going from bulk to nanofilm appears to be a general phenomenon that has been predicted to occur for a number of materials. ${ }^{43}$ The new nanofilms NF1-NF4 found in our SMA searches all have energies slightly higher than the bixbyite nanofilm by $5.5-26.2 \mathrm{~kJ} \mathrm{~mol}^{-1}$ per $\mathrm{Ce}_{2} \mathrm{O}_{3}$. It is of note that the four nanofilms, NF1-NF4, have structures which do not correspond to any known bulk crystalline $\mathrm{A}_{2} \mathrm{O}_{3}$ polymorph. The 4 ML NF1 nanofilm is particularly interesting as it is the only new film that is predicted to be more energetically stable than the A-type $4 \mathrm{ML}$ nanofilm. We note that this prediction is also confirmed by our calculations using the hybrid HSE06 functional. In Fig. 2 we show the structures of the NF1, bixbyite and A-type $4 \mathrm{ML}$ nanofilms. For these three nanofilms we have performed GGA $+U$ calculations under externally applied stress or strain (within the plane of each nanofilm) and gener-

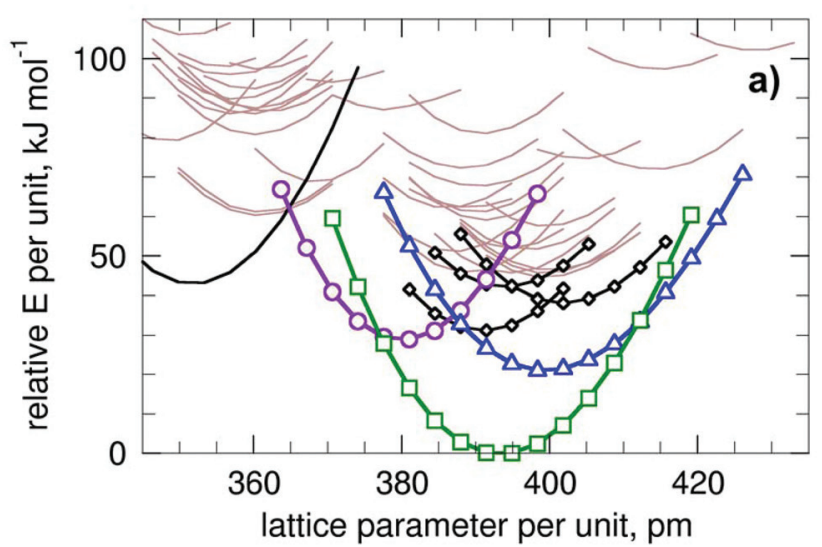

$\mathrm{Cu}(111) \mathrm{Si}(111) \mathrm{CeO}_{2}(111) \operatorname{Rh}(111) \mathrm{Re}(0001) \mathrm{Pt}(111)$

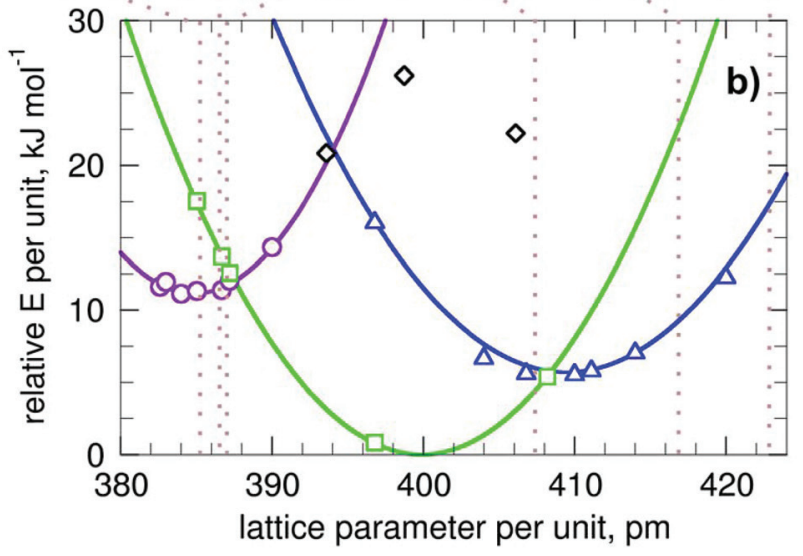

Fig. 1 Results of (a) the IP-based SMA search, and (b) GGA + U calculations for films with: A-type (circles), bixbyite (squares) and NF1 (triangles), NF2-4 (diamonds), corundum (black, no symbol) and other structures (brown, no symbol). Energies (relative to that of the optimized bixbyite nanofilm) and lattice parameters are given per $\mathrm{Ce}_{2} \mathrm{O}_{3}$ unit. Solid lines in (b) are parabolic fits to the data points to guide the eye. Vertical dotted lines in (b) indicate GGA-calculated lattice parameters of possible supports for nanofilm growth (multiplied by $3 / 2$ for transition metals highlighted by italics).

ated three characteristic curves of relative energy versus the inplane $a$ lattice parameter per $\mathrm{Ce}_{2} \mathrm{O}_{3}$ unit (see Fig. 1b). The shapes and relative positions of the three curves in Fig. 1b match quite well with the corresponding IP-based curves (see highlighted curves in Fig. 1a). Although the DFT-calculated energetic ordering of the nanofilms is generally well reproduced by the IP calculations, the latter results span a twice larger energy range. This finding is fully in line with a combined IP and GGA $+U$ study of partially reduced ceria nanoclusters. ${ }^{26}$ We note that according to the IP data bixbyite films are more stable than A-type and NF1 films even at their points of minimum energy. For the DFT calculations, however, the NF1 and A-type energy minima lay outside of the energy versus strain curve of bixbyite. This finding suggests that by using substrates with different lattice parameters, one could favour the epitaxial growth of a particular nanofilm structure.

Experimentally, a few $\mathrm{Ce}_{2} \mathrm{O}_{3}$ nanofilm structures have already been produced on different substrates. In Fig. 1b we 
A-type
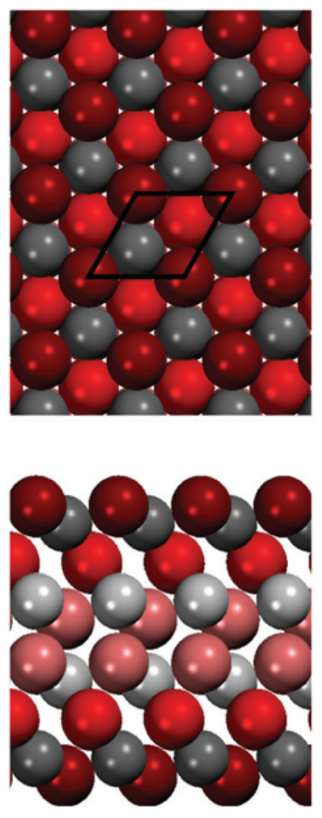

bixbyite
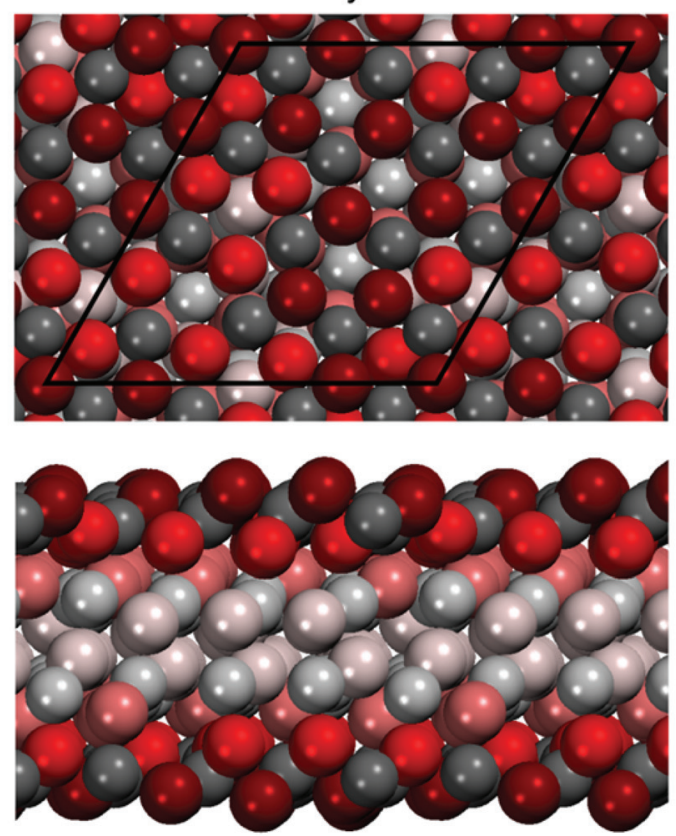

NF1
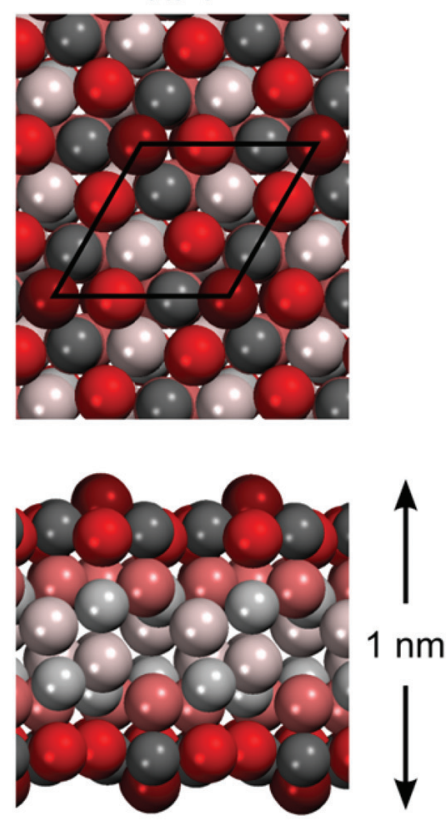

Fig. 2 Top and side views of A-type, bixbyite and $\mathrm{NF}_{1} \mathrm{Ce}_{2} \mathrm{O}_{3}$ nanofilms of $4 \mathrm{ML}$ thickness. $\mathrm{O}$ atoms are displayed as red spheres and Ce ${ }^{3+}$ ions as grey spheres. Atoms with darker colors are located in surface layers. Employed unit cells are denoted by black lines.

include the in-plane lattice parameters of a selection of surfaces that have been employed to grow supported $\mathrm{Ce}_{2} \mathrm{O}_{3}$ nanofilms, as calculated using the GGA $+U$ method. For the $\mathrm{Cu}(111)$ surface, $2.5 \mathrm{ML}$ fluorite $\mathrm{CeO}_{2}(111)$ nanofilms were grown with a $2: 3$ epitaxy. Upon heating to $1070 \mathrm{~K}$ these nanofilms could be transformed into $\mathrm{Ce}_{2} \mathrm{O}_{3}$ nanofilms with the A-type structure while retaining a very similar epitaxial matching. ${ }^{44}$ From a thermodynamical perspective, such a transition is in agreement with our calculations (Fig. 1b) where the $\mathrm{Cu}(111)$ surface and A-type films have closely matching lattice parameters (after multiplying the lattice parameter of $\mathrm{Cu}(111)$ by 3/2). Using metallic Ce as a reducing agent, and annealing under slightly milder thermal conditions $(900 \mathrm{~K})$, similar $\mathrm{Cu}$ (111)-supported $4 \mathrm{ML} \mathrm{CeO}_{2}$ films could be reduced to $\mathrm{Ce}_{2} \mathrm{O}_{3}$ nanofilms exhibiting the bixbyite structure. ${ }^{9}$ Here, assuming there is no structural relaxation of the $\mathrm{Cu}(111)$ surface and perfect $3: 2$ epitaxy, we predict that a suitably contracted freestanding $4 \mathrm{ML}$ bixbyite nanofilm would be moderately metastable $\left(+6 \mathrm{~kJ} \mathrm{~mol}^{-1}\right.$ per $\mathrm{Ce}_{2} \mathrm{O}_{3}$ ) relative to an A-type nanofilm with the same lattice parameter (see Fig. 1b). We thus suggest that the observation of bixbyite films grown at relatively moderate temperatures on $\mathrm{Cu}(111)$ does not necessarily require their preferential energetic stability on the support. Rather, it can be due to kinetics whereby the preparation retains much of the original fluorite structure of the $\mathrm{CeO}_{2}$ precursor. Bixbyite $\mathrm{Ce}_{2} \mathrm{O}_{3}$ nanofilms of 2-5 ML have also been grown on Clpassivated $\mathrm{Si}(111)$ surfaces by Flege $e t$ al. ${ }^{8}$ For such a situation we predict an even smaller metastability of $4 \mathrm{ML}$ bixbyite films $\left(+2 \mathrm{~kJ} \mathrm{~mol}^{-1}\right.$ per $\left.\mathrm{Ce}_{2} \mathrm{O}_{3}\right)$ with respect to A-type. This very small calculated energy difference points again to kinetic stabilization of these experimentally observed bixbyite nanofilms.
For the significantly larger lattice parameter of $\mathrm{Rh}(111)$, supported $\mathrm{CeO}_{2}$ nanofilms with 1-6 ML thicknesses have been shown to decompose at temperatures of $700-800{ }^{\circ} \mathrm{C}$ to give reduced ceria islands and a $(4 \times 4)$ Low-Energy Electron Diffraction (LEED) pattern. ${ }^{45}$ Although in ref. 45 this LEED pattern is ascribed to Ce-Rh alloy formation, with hindsight, another interpretation of such a measurement may be the emergence of the bixbyite structure. In Fig. $1 \mathrm{~b}$ we see that such an interpretation is consistent with the calculated small energetic preference for $3: 2$ epitaxial 4 ML bixbyite nanofilms on $\mathrm{Rh}(111)$.

Although we are aware of no reports directly identifying our predicted NF1 nanofilm, we can see from Fig. 1b that supports with a larger lattice parameter than those cited above for ultrathin films would be required to produce NF1, for instance, $\operatorname{Re}(0001)$ or $\mathrm{Pt}(111)$ with calculated $a_{0}$ of 278 and $282 \mathrm{pm}$, respectively. In fact, reduced ceria films have been prepared on $\operatorname{Re}(0001),{ }^{46}$ but, as far as we are aware, only with relatively large thicknesses $(>20 \mathrm{ML})$ of limited relevance to the present study. On the Pt(111) surface, reduction of 1-2 $\mathrm{ML} \mathrm{CeO}_{2}$ nanofilms with $4: 3$ epitaxy has led to novel nanofilms with, as yet, undetermined structures. ${ }^{47,48}$ Assuming a $3: 2$ epitaxy, our calculations indicate that the $\mathrm{Pt}(111)$ surface should thermodynamically favour the formation of the NF1 nanofilm relative to bixbyite and A-type. In ref. 47 a strongly reduced $2 \mathrm{ML} \mathrm{CeO}_{2}$ nanofilm is found to exhibit an unresolved structure with a $9 / 4(\sqrt{ } 3 \times \sqrt{ } 3) R 30^{\circ}$ periodicity (with respect to Pt) which is consistent with that of NF1 (see Fig. 2). Similarly to the structure of NF1, the $1 \mathrm{ML} \mathrm{Ce}_{2} \mathrm{O}_{3}$ nanofilm reported in ref. 48 has a hexagonal unit cell with a lattice constant that is approximately twice that of A-type (see Table 1). Additionally, scanning 
Table 2 Calculated GGA $+U$ energy gap values $\Delta \varepsilon$ (in eV) between the highest occupied $(\mathrm{HO})$ and the lowest unoccupied (LU) states of $\mathrm{Ce}$ and $\mathrm{O}$ and average coordination numbers of $\mathrm{Ce}, \mathrm{N}(\mathrm{Ce})$, in bulk and $4 \mathrm{ML}$ nanofilm structures ${ }^{a}$

\begin{tabular}{lllll}
\hline System & $\Delta \varepsilon\left(\mathrm{HO}_{\mathrm{Ce}}-\mathrm{HO}_{\mathrm{O}}\right)$ & $\Delta \varepsilon\left(\mathrm{HO}_{\mathrm{Ce}}-\mathrm{LU}_{\mathrm{O}}\right)$ & $\Delta \varepsilon\left(\mathrm{HO}_{\mathrm{O}}-\mathrm{LU}_{\mathrm{Ce}}\right)$ & $\mathrm{N}(\mathrm{Ce})^{b}$ \\
\hline A-type bulk & 1.4 & 2.0 & 3.9 & 7.00 \\
Bixbyite bulk & 1.8 & 1.7 & 3.8 & 6.00 \\
& & & & \\
A-type film & 1.4 & 2.3 & 4.1 & 6.50 \\
Bixbyite film & 1.1 & 0.8 & 2.7 & 5.63 \\
NF1 film & 1.4 & 1.0 & 2.7 & 5.50
\end{tabular}

${ }^{a}$ Note that the presented GGA $+U$ absolute band gap values $\Delta \varepsilon\left(\mathrm{HO}_{\mathrm{O}^{-}}\right.$ $\mathrm{LU}_{\mathrm{Ce}}$ ) are expected to be notably underestimated with respect to both hybrid-functional DFT calculations and experimental data. ${ }^{3}{ }^{b}$ Average coordination numbers of $\mathrm{O}$ are 1.5 times smaller than $\mathrm{N}(\mathrm{Ce})$.

tunneling microscopy of this latter nanofilm shows protruding adatoms at three-fold coordinated sites. This observation is in accordance with the curious structure of NF1, which displays protruding oxygen atoms at three-fold coordinated sites, albeit with a higher density than that observed in the experiment. The finding that the adatoms in the experimentally prepared $1 \mathrm{ML}$ nanofilm are disordered whereas those in NF1 are ordered may be a reflection of experimental conditions (e.g. finite temperatures, $1 \mathrm{ML}$ versus $4 \mathrm{ML}$ ) or again kinetic limitations.

In order to encourage further experimental work to better characterize such reduced ceria nanofilms, in Table 2 we present some calculated properties of NF1 to help distinguish them from A-type and bixbyite nanofilms. Firstly, in line with its relatively larger in-plane lattice parameter, both the Ce and $\mathrm{O}$ atoms in the NF1 nanofilm have lower average coordination numbers than in A-type and bixbyite nanofilms. Secondly, with respect to electronic properties, GGA $+U$ band gaps $(\mathrm{O} 2 \mathrm{p}-\mathrm{Ce} 4 \mathrm{f}+5 \mathrm{~d})$ are rather similar, $\sim 3.9 \mathrm{eV}$, in the considered bulk structures and A-type film. However, in bixbyite and NF1 films these band gaps are reduced to $\sim 2.7 \mathrm{eV}$, which could be explained by the presence of five-coordinated Ce ions. In fact, under-coordinated Ce ions have been already shown to reduce the band gap in $\mathrm{CeO}_{2}$ nanoparticles and concomitantly greatly increase their reducibility. ${ }^{49}$ As these properties are amenable to measurement (e.g. via EXAFS, PES), we hope that our predicted new NF1 nanofilm will be identified in future experimental studies.

\section{Conclusions}

To summarise, using simulated mechanical annealing searches and density functional calculations we identify a range of new low energy $4 \mathrm{ML} \mathrm{Ce}_{2} \mathrm{O}_{3}$ nanofilm structures. We find that our calculations of energetic stability versus in-plane lattice parameter are consistent with the stability of experimentally observed nanofilm phases depending on the substrates used to prepare them. Further, we propose a new energetically stable NF1 film structure and suggest suitable substrates that would favor its growth. We note that our predicted NF1 nanofilm appears to have some structural properties consistent with those reported for reduced ceria films on $\mathrm{Pt}(111)$ surfaces. Finally, we present specific calculated properties of the NF1 nanofilm that should assist in its experimental identification.

\section{Acknowledgements}

SMK thanks the Spanish MEDU for FPU grant AP2009-3379. This study has been supported by grants of the Spanish Ministry of Economy and Competitiveness (CTQ2012-34969; MAT2012-30924), the European Union FP7 Program under grant agreement number 310191, the Generalitat de Catalunya (2014SGR97; XRQTC) and by the COST Action CM1104. We also acknowledge the use of supercomputing resources provided by the Red Española de Supercomputación.

\section{References}

1 S. T. Bromley, I. de P. R. Moreira, K. M. Neyman and F. Illas, Chem. Soc. Rev., 2009, 38, 2657-2670.

2 Catalysis by Ceria and Related Materials, ed. A. Trovarelli and P. Fornasiero, Imperial College Press, London, 2nd edn, 2013.

3 J. Paier, C. Penschke and J. Sauer, Chem. Rev., 2013, 113, 3949-3985.

4 A. Migani, G. N. Vayssilov, S. T. Bromley, F. Illas and K. M. Neyman, Chem. Commun., 2010, 46, 5936-5938.

5 A. Migani, G. N. Vayssilov, S. T. Bromley, F. Illas and K. M. Neyman, J. Mater. Chem., 2010, 20, 10535-10546.

6 S. M. Kozlov and K. M. Neyman, Phys. Chem. Chem. Phys., 2014, 16, 7823-7829.

7 S. Carrettin, P. Concepción, A. Corma, J. M. López Nieto and V. F. Puntes, Angew. Chem., Int. Ed., 2004, 43, 2538-2540.

8 J. I. Flege, B. Kaemena, S. Gevers, F. Bertram, T. Wilkens, D. Bruns, J. Bätjer, T. Schmidt, J. Wollschläger and J. Falta, Phys. Rev. B: Condens. Matter, 2011, 84, 235418.

9 V. Stetsovych, F. Pagliuca, F. Dvořák, T. Duchoň, M. Vorokhta, M. Aulická, J. Lachnitt, S. Schernich, I. Matolínová, K. Veltruská, T. Skála, D. Mazur, J. Mysliveček, J. Libuda and V. Matolín, J. Phys. Chem. Lett., 2013, 4, 866-871.

10 Oxide Ultrathin Films: Science and Technology, ed. G. Pacchioni and S. Valeri, Wiley-VCH, Weinheim, 2011.

11 G. Kresse, M. Schmid, E. Napetschnig, M. Shishkin, L. Köhler and P. Varga, Science, 2005, 308, 1440-1442.

12 J. Weissenrieder, S. Kaya, J.-L. Lu, H.-J. Gao, S. Shaikhutdinov, H.-J. Freund, M. Sierka, T. K. Todorova and J. Sauer, Phys. Rev. Lett., 2005, 95, 076103.

13 D. Löffler, J. J. Uhlrich, M. Baron, B. Yang, X. Yu, L. Lichtenstein, L. Heinke, C. Büchner, M. Heyde, S. Shaikhutdinov, H.-J. Freund, R. Włodarczyk, M. Sierka and J. Sauer, Phys. Rev. Lett., 2010, 105, 146104. 
14 M. Kiguchi, S. Entani, K. Saiki, T. Goto and A. Koma, Phys. Rev. B: Condens. Matter, 2003, 68, 115402.

15 C. Tusche, H. L. Mayerheim and J. Kirschner, Phys. Rev. Lett., 2007, 99, 026102.

16 J. Goniakowski, C. Noguera and L. Giordano, Phys. Rev. Lett., 2004, 93, 215702.

17 C. L. Freeman, F. Claeyssens, N. L. Allan and J. H. Harding, Phys. Rev. Lett., 2006, 96, 066102.

18 S. Tosoni, B. Civalleri and P. Ugliengo, J. Phys. Chem. C, 2010, 114, 19984-19992.

19 A. Navrotsky, Proc. Natl. Acad. Sci. U. S. A., 2004, 101, 12096-12101.

20 W. Sangthong, J. Limtrakul, F. Illas and S. T. Bromley, Nanoscale, 2010, 2, 72-77.

21 N. Krainara, J. Limtrakul, F. Illas and S. T. Bromley, Phys. Rev. B: Condens. Matter, 2011, 83, 233305.

22 N. Krainara, J. Limtrakul, F. Illas and S. T. Bromley, J. Phys. Chem. C, 2013, 117, 22908-22914.

23 Z. W. Shan, R. K. Mishra, S. A. Syed Asif, O. L. Warren and A. M. Minor, Nat. Mater., 2007, 7, 115-119.

24 T. X. T. Sayle, S. C. Parker and C. R. A. Catlow, J. Chem. Soc., Chem. Commun., 1992, 977-978.

25 J. C. Conesa, Surf. Sci., 1995, 339, 337-352.

26 A. Migani, K. M. Neyman, F. Illas and S. T. Bromley, J. Chem. Phys., 2009, 131, 064701.

27 J. D. Gale, Z. Kristallogr., 2005, 220, 552-554.

28 A. Migani, K. M. Neyman and S. T. Bromley, Chem. Commun., 2012, 48, 4199-4201.

29 J. P. Perdew and Y. Wang, Phys. Rev. B: Condens. Matter, 1992, 45, 13244-13249.

30 G. Kresse and J. Hafner, Phys. Rev. B: Condens. Matter, 1993, 47, 558-561.

31 V. I. Anisimov, F. Aryasetiawan and A. I. Lichtenstein, J. Phys.: Condens. Matter, 1997, 9, 767-808.

32 S. L. Dudarev, G. A. Botton, S. Y. Savrasov, C. J. Humphreys and A. P. Sutton, Phys. Rev. B: Condens. Matter, 1998, 57, 1505-1509.
33 J. L. F. Da Silva, Phys. Rev. B: Condens. Matter, 2007, 76, 193108.

34 P. E. Blöchl, Phys. Rev. B: Condens. Matter, 1994, 50, 1795317979.

35 G. Kresse and D. Joubert, Phys. Rev. B: Condens. Matter, 1999, 59, 1758-1775.

36 H. J. Monkhorst and J. D. Pack, Phys. Rev. B: Condens. Matter, 1976, 13, 5188-5192.

37 H. Bärnighausen and G. Schiller, J. Less-Common Met., 1985, 110, 385-390.

38 H. Jiang, P. Rinke and M. Scheffler, Phys. Rev. B: Condens. Matter, 2012, 86, 125115.

39 As an example, we note that the energy associated with improper f-orbital symmetry can be up to $\sim 0.5 \mathrm{eV}$ per $\mathrm{Ce}_{2} \mathrm{O}_{3}$, when the symmetry of all orbitals in the cell are erroneous.

40 D. R. Hamann, Phys. Rev. B: Condens. Matter, 1996, 76, 660-663.

41 J. Kang, E.-C. Lee and K. J. Chang, Phys. Rev. B: Condens. Matter, 2003, 68, 054106.

42 J. Heyd, G. E. Scuseria and M. Ernzerhof, J. Chem. Phys., 2003, 118, 8207-8215.

43 I. Demiroglu and S. T. Bromley, Phys. Rev. Lett., 2013, 110, 245501.

44 V. Matolín, J. Libra, I. Matolínová, V. Nehasil, L. Sedláček and F. Šutara, Appl. Surf. Sci., 2007, 254, 153-155.

45 S. Eck, C. Castellarin, S. Surnev, M. G. Ramsey and F. P. Netzer, Surf. Sci., 2002, 520, 173-185.

46 W. Xiao, Q. Guo and E. G. Wang, Chem. Phys. Lett., 2003, 368, 527-531.

47 P. Luches, F. Pagliuca and S. Valeri, Phys. Chem. Chem. Phys., 2014, 16, 18848-18857.

48 U. Berner and K.-D. Schierbaum, Phys. Rev. B: Condens. Matter, 2002, 65, 235404.

49 M. A. Sk, S. M. Kozlov, K. H. Lim, A. Migani and K. M. Neyman, J. Mater. Chem. A, 2014, 2, 1832918338. 